\title{
Capacitance Online Estimation Based on Adaptive Model Observer
}

\author{
Zhaohui Cen \\ Qatar Environment and Energy Research Institute, HBKU, Qatar Foundation, 5825, Doha, Qatar
}

\begin{abstract}
As a basic component in electrical and electronic devices, capacitors are very popular in electrical circuits. Conventional capacitors such as electrotype capacitors are easy to degradation, aging and fatigue due to long-time running and outer damages such as mechanical and electrical stresses. In this paper, a novel online capacitance measurement/estimation approach is proposed. Firstly, an Adaptive Model Observer (AMO) is designed based on the capacitor's circuit equations. Secondly, the AMO's stability and convergence are analysed and discussed. Finally, Capacitors with different capacitance and different initial voltages in a buck converter topology are tested and validated. Simulation results demonstrate the effectiveness and superiority of our proposed approach.
\end{abstract}

\section{Introduction}

Due to their penetration in electrical and electronic circuits, capacitors especially super-capacitors play a key role in information technologies and electrical engineering. There are many types of capacitors in the market, their conductors may be thin films, foils or sintered beads of metal or conductive electrolyte, etc. Also, the capacitors will be used in different application scenarios based on different capabilities such as filtering and energy storage. Because of its manufacturing drawbacks, long-time running and outer damages such as mechanical and electrical stresses, capacitors are easy to degrade and fatigue, which will cause the rated capacitance vary and probably cause failure for overall system[1], [2]. For example, many computers cannot be restarted because the capacitors in the mainboard are damaged in a form of capacitor plague. In some cases, the capacitors may cause an exploration due to the electrolyte chemistry reaction, which is a possible cause for some disasters. Therefore, it is necessary to monitoring the capacitor's status and conditions in realtime in some applications, especially power system applications such as power converters. Regarding capacitor's condition parameters, mostly used parameter is the measured capacitance value because the measured capacitance can directly indicate whether a capacitor is working in the rated capacitance, and how much the capacitance variation is because of degradation and aging.

Currently, there are few approaches to measure and monitor the capacitors' parameters and conditions. The measurement parameters mainly include capacitance, internal resistance and impedances, where capacitance is mostly adopted to measure and evaluate the status of a capacitor [3]. Regarding capacitance measurement approaches, we can classify existed methods into two classifies: offline measurement and online measurement [4]-[8]. The off-line measurement is easy to implement because most multi-meters can measure the capacitance by touching two pins of a capacitor, the drawbacks of this measurement is that, the capacitor need to be off-line and even uninstalled from the board, because the multi-meter need to use its self-generated stimulating signal to acquire and calculate the capacitance. In some improved off-line approach (may be called as quasi-online method), the offline and online mode may be switched in a short time by the test logic control in order to compute the capacitance, but actually it is still not the best solution because the capacitor still need to be offline more or less, which is not applicable in some power applications. Aimed at the offline method drawbacks, some online capacitance estimation methods are proposed to estimate the capacitance of capacitors in wind turbo applications [9]. By injecting stimulating signal at some specified frequencies into power source or voltage of the capacitor, the capacitance can be estimated based on the response of capacitor voltage in the specified frequency. The drawback of this approach is that, the operation is very complicated, and also how to set frequency for capacitors with different capacitance and how to filtering the voltage signal is not clear for a real-world applications. Actually, the best desirable approach is that we don't need to change anything for the test capacitor and keep it online for capacitance estimation based on existed measurement signal and devices such as voltage and current sensors.

Motived from the demand in this regarding, the main contribution of this paper is that a novel adaptive model observer method is proposed to address the online capacitance estimation challenges. Compared with existed capacitance measurement approaches, the proposed approach has an obvious advantage: it can keep 
the test capacitor online and only need the voltage and current signals from the capacitor pins.

\section{Problem to be addressed}

In this paper, we will address the capacitance drafting problem. We use a typical case of capacitance shown in Fig. 1. The rated capacitance in the capacitor is $1000 \mathrm{uF}$, but the actual capacitance is $839.2 \mathrm{uF}$, which means the capacitance drafting is around $15 \%$.

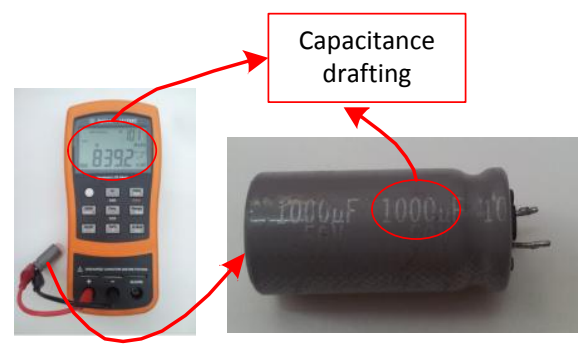

Figure 1. Capacitance drafting

So, it is needed to monitor the capacitance in realtime and online conditions. For capacitors, they can be generally denoted as its Current-voltage relation equation as following.

$$
I(\mathrm{t})=\frac{d Q(\mathrm{t})}{d t}=C \frac{d V(\mathrm{t})}{d t}
$$

For measurement, the capacitor voltage $V(\mathrm{t})$ and current $I(\mathrm{t})$ are available variables by senor online measurement. The problem is how to estimate the $C$ of (1) in real-time.

\section{Capacitance estimation scheme based on adaptive model observer}

In order to estimate the capacitance in real-time, we designed a special adaptive observer to tracking and estimate the state and capacitance.

\subsection{Adaptive model observer}

Based on the current-voltage relationship in (1), we propose an adaptive model observer in the format as following.

$$
C \frac{d \hat{V}(\mathrm{t})}{d t}-I(t)-K(V(t)-\hat{V}(t))=0
$$

where $K$ is the observer feedback gain. If we select the proper $K$, the observer state will follow as $V(t)-\hat{V}(t) \rightarrow 0$.

So, based on convergence principle as shown above, a capacitance estimation scheme can be derived as following.

$$
\begin{aligned}
& \hat{C} \frac{d \hat{V}(\mathrm{t})}{d t}-I(t)=0 \\
& \hat{C}=K(V(t)-\hat{V}(t))
\end{aligned}
$$

By the feedback control from $K$, the capacitance can be estimated based on available variables such as the capacitor voltage $V(\mathrm{t})$ and current $I(\mathrm{t})$

\subsection{Online estimation scheme}

The online estimation scheme based on (3) can be shown in Fig. 2. Fig. 2 depicts the capacitance estimation scheme for a general capacitor, which can be used for real-time monitoring for any circuit topologies once voltage and current measurement is available. As can be seen from Fig. 1, the capacitor voltage and current need to be measured and sent into DSP controller for processing. The adaptive model observer estimates the online capacitance based on the observer feedback loop and adaptive estimation law. In this scheme, different initial

Capacitance under different initial value of the integer $1 / \mathrm{s}$ will converge into the estimated capacitance under the adaptive estimation law.

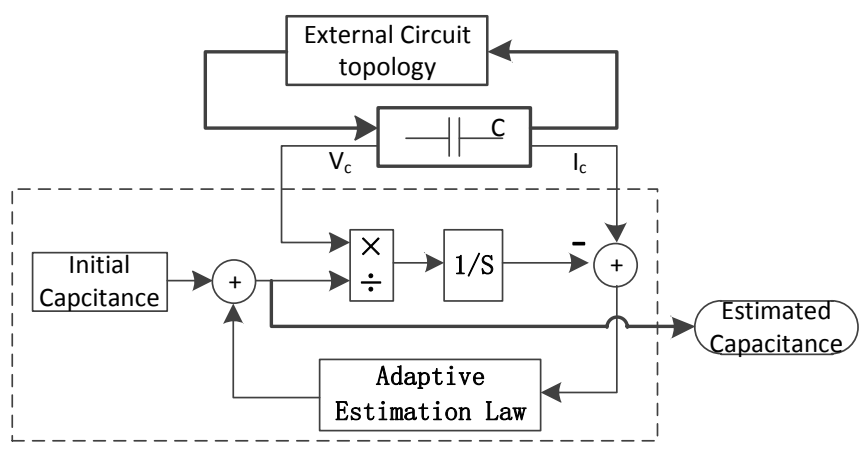

Figure 2. Online estimation scheme for general capacitors

\section{Simulation results}

In order to validate the effectiveness of our proposed method, we use a typical converter topology which include two capacitors (Shown in Fig. 2) to validate our proposed scheme. In this topology, two capacitors $\mathrm{C} 1$ $(350 \mathrm{uF})$ and $\mathrm{C} 2(100 \mathrm{uF})$ are used for stabilize the input voltage and output voltage respectively.

The capacitance online estimation result of the capacitor $\mathrm{C} 1$ and $\mathrm{C} 2$ can be seen in Fig. 4 and Fig. 3 respectively. As can be seen from Fig. 4 (a) and (b), the capacitor voltage and current are variable with time and the transient characteristics are very obvious. As can be from Fig. 4 (c), the voltage residual can converge very quickly under the feedback gain control. As can be seen from Fig. 4(d), the estimation can track the real capacitance in a fast speed. Fig. 5 shows the online estimation result for capacitor $\mathrm{C} 2$. As can be seen from Fig. 5 (a) and (b), although the voltage and current is stable and different from $\mathrm{C} 1$, the online estimation scheme can also track the capacitance value $100 \mathrm{uF}$ very quickly. 


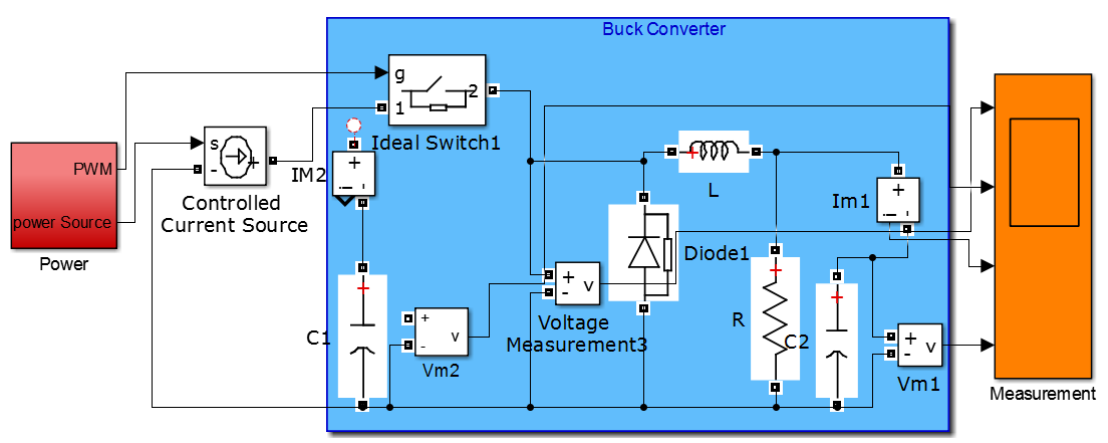

Figure 3. Test Buck converter topology

(a)

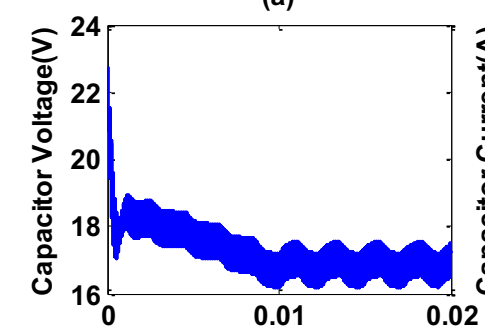

(c)

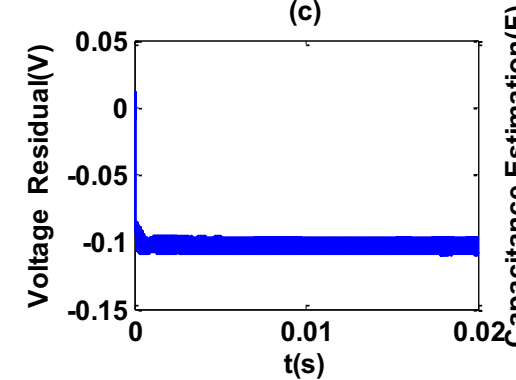

$\mathrm{t}(\mathbf{s})$ (b)

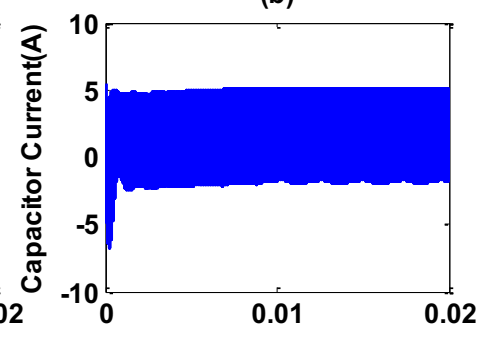

(d)

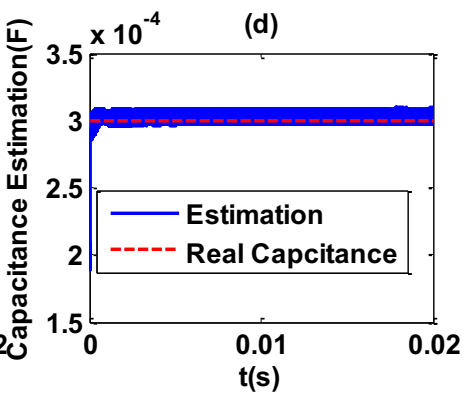

Figure 4. Online estimation result of the Capacitor $\mathrm{C} 1$

(a)

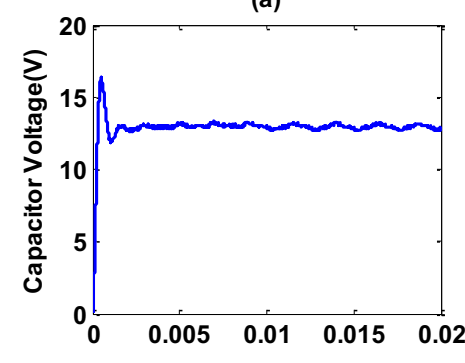

(c)

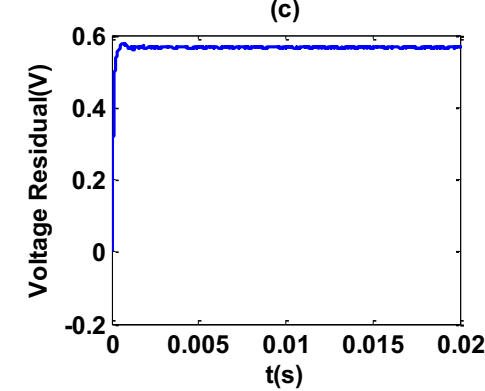

Figure 5. Online estimation results of the Capacitor C

\section{Conclusions}

In this paper, a novel capacitance estimation scheme is proposed to monitor capacitor $\mathrm{s}$ and estimate their
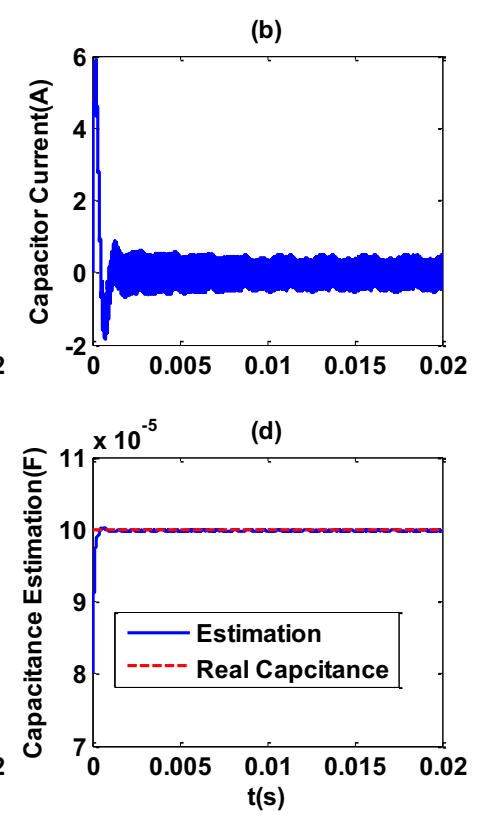

capacitance. A buck converter consisting of two capacitors are employed to test and validate the effectiveness of the proposed scheme. Simulation results demonstrate that the capacitance online estimation scheme can track the real capacitance in a quick speed. Also, this scheme is applicable for capacitors in any 
topology no matter that it is online. Compared with existed approaches, our approach is more general for any capacitors and implements real-time monitoring. Our future work will focus on experiment demonstration and also consider the internal resistance's effects.

\section{References}

1. A. M. Imam, T. G. Habetler, R. G. Harley, and D. M. Divan, "Condition monitoring of electrolytic capacitor in power electronic circuits using adaptive filter modeling," in 2005 IEEE 36th Power Electronics Specialists Conference, (2005).

2. D.-C. Lee, K.-J. Lee, J.-K. Seok, and J.-W. Choi, "Online capacitance estimation of DC-link electrolytic capacitors for three-phase $\mathrm{AC} / \mathrm{DC} / \mathrm{AC}$ PWM converters using recursive least squares method," in Electric Power Applications, IEE Proceedings-, 152, no. 6, pp. 1503-1508, (2005).

3. C. Kulkarni, G. Biswas, X. Koutsoukos, J. Celaya, and K. Goebel, "Integrated diagnostic/prognostic experimental setup for capacitor degradation and health monitoring," in AUTOTESTCON, 2010 IEEE, pp. 1-7, (2010).

4. G. Yifeng, "Analysis and design of the super capacitor monitoring system of hybrid electric vehicles," Procedia Eng., 15, pp. 90-94, (2011).

5. P. XUE, J. GAO, Y. ZHANG, X. WANG, and G. WANG, "Discussion about the Way to Measure Capacitance and dielectric Loss of Circuit Breaker Capacitor [J]," Power Capacit., 4, (2007).

6. B. R. Lee, "Method for monitoring the capacitance of a capacitor while adjusting its capacitance." Google Patents, 30-May-1972.

7. R. A. Kumar, M. S. Suresh, and J. Nagaraju, "Time domain technique to measure solar cell capacitance," Rev. Sci. Instrum., 74, no. 7, pp. 3516-3519, (2003).

8. A. D. Kompelien, "Capacitance monitoring bridge circuit for an enthalpy responsive device." Google Patents, Dec. 17, (1985).

9. A. G. Abo-Khalil and D.-C. Lee, "DC-link capacitance estimation in AC/DC/AC PWM converters using voltage injection," Ind. Appl. IEEE Trans., 44, no. 5, pp. 1631-1637, (2008). 